\title{
VUV synchrotron radiation processing of thin palladium acetate spin-on films for metallic surface patterning
}

\author{
Y. Zhang and M. Stuke \\ Max-Planck-Institut für biophysikalische Chemie, Postfach 2841, D-3400 Göttingen, Fed. Rep. of Germany
}

Received 29 May 1990; accepted for publication 26 July 1990

Processing of thin palladium acetate spin-on films for the electroless copper plating has been carried out using UV and VUV synchrotron radiation. The mechanism of UV photodeposition of palladium from the thin palladium acetate film is studied.

\section{Introduction}

Thin palladium acetate films have been used for laser deposition (or prenucleation) of palladium lines, which can be used as a catalyst for electroless plating of other metals, notably $\mathrm{Cu}$, to build up thicker conductors. This palladium deposition process was carried out using a CW argon ion laser at $514.5 \mathrm{~nm}$ for the first time in 1987 [1] then at $351 \mathrm{~nm}[2]$. This visible-laser-induced deposition of palladium is a photothermal process since the Pd-acetate film precursor has no significant electronic absorption above $350 \mathrm{~nm}$ (see spectrum (a) in fig. 3). Powerful visible laser radiation has to be used to heat up the absorbing area of a substrate to initiate a deposition and therefore, this process cannot be carried out on a transparent substrate like quartz. Further, UV excimer laser (ArF at $193 \mathrm{~nm}, \mathrm{KrF}$ at $248 \mathrm{~nm}$ or $\mathrm{XeCl}$ at $308 \mathrm{~nm}$ ) was used for the same processing [3] since the film precursor has strong UV absorption at about $210 \mathrm{~nm}$ (see spectrum (a) in fig. 3). Although the processing with the UV excimer laser pulses was carried out on UV transparent substrate like quartz, it was still doubtful whether this process is photolytic or photothermal when the laser fluence threshold for prenucleation had been found for each UV excimer laser wavelength (see
Table 1

Photodeposition of palladium from Pd-acetate spin-on films

\begin{tabular}{|c|c|c|c|}
\hline \multirow[t]{3}{*}{ Visible } & $\mathrm{Ar}^{+}(514.5 \mathrm{~nm})$ & $\begin{array}{l}10^{5} \mathrm{~W} \\
\mathrm{~cm}^{-2}\end{array}$ & Gross et al. [1] \\
\hline & $\mathrm{Ar}^{+}(351 \mathrm{~nm})$ & $\begin{array}{l}10^{4} \mathrm{w} \\
\mathrm{cm}^{-2}\end{array}$ & Cole et al. [2] \\
\hline & $\mathrm{XeF}(351 \mathrm{~nm})$ & $\begin{array}{c}>200 \mathrm{~mJ} \\
\mathrm{~cm}^{-2}\end{array}$ & Esrom and Wahl [3] \\
\hline \multirow[t]{4}{*}{ UV } & $\mathrm{CeCl}(308 \mathrm{~nm})$ & $\begin{array}{c}>80 \mathrm{~mJ} \\
\mathrm{~cm}^{-2}\end{array}$ & Esrom and Wahl [3] \\
\hline & $\mathrm{KrF}(248 \mathrm{~nm})$ & $\begin{aligned} &> 15 \mathrm{~mJ} \\
& \mathrm{~cm}^{-2}\end{aligned}$ & \\
\hline & $\operatorname{ArF}(193 \mathrm{~nm})$ & $\begin{array}{r}>10 \mathrm{~mJ} \\
\mathrm{~cm}^{-2}\end{array}$ & \\
\hline & Synchrotron & $\begin{array}{c}<0.6 \mathrm{~mW} \\
\mathrm{~cm}^{-2}\end{array}$ & This work \\
\hline VUV & Synchrotron & $\begin{array}{c}<25 \mathrm{~mW} \\
\mathrm{~cm}^{-2}\end{array}$ & This work \\
\hline
\end{tabular}

table 1). In order to clarify this, we studied the UV photochemistry of $\mathrm{Pd}$-acetate spin-on film. The result of this study is in agreement with our successful experiment of deposition of palladium from Pd-acetate films using VUV synchrotron radiation with an average flux as low as $25 \mathrm{~mW}$ $\mathrm{cm}^{-2}$. A comparison of the photodeposition of palladium from Pd-acetate spin-on films using different light sources is given in table 1. 


\section{Experiment}

The palladium acetate $\left(\mathrm{Pd}\left(\mu-\mathrm{O}_{2} \mathrm{CCH}_{3}\right)_{2}\right)_{3}$ was spin-coated from a chloroform solution onto different substrates $\left(\mathrm{Al}_{2} \mathrm{O}_{3}\right.$ ceramic, quartz and silicon wafer) to a thickness ranging from 0.02 to 1.5 $\mu \mathrm{m}$, depending on solution concentration and spin speed. The thickness of the spin-on film was measured using a Sloan Dektak IIA stylus profilometer.

UV spectra of the palladium acetate film on a quartz substrate were recorded on a Shimadzu UV-160 spectrometer, which was also used to estimate the thickness of the thin film below 0.05 $\mu \mathrm{m}$. IR spectra of the palladium acetate films on either a silicon wafer or an $\mathrm{Al}_{2} \mathrm{O}_{3}$ ceramic were recorded on a Nicolet 740 SX Fourier transform infrared spectrometer to monitor the chemical change inside the palladium acetate film exposed to UV laser light. A Balzers QMG 511 quadrupole mass spectrometer was used to detect desorbed species during the UV excimer laser irradiation of the Pd-acetate film under high vacuum.

The UV or VUV light source was taken either from a Lambda Physik EMG 500 excimer laser at
$248 \mathrm{~nm}(\mathrm{KrF})$ or $193 \mathrm{~nm}(\mathrm{ArF})$, or from the synchrotron radiation provided by the electron storage ring (BESSY) in Berlin, which, through a Wadsworth monochromator without being dispersed (at the zeroth order of the grating), gives a 40-300 $\mathrm{nm}$ VUV beam of an average flux of $5 \times 10^{15}$ photons $\mathrm{s}^{-1} \mathrm{~cm}^{-2}$ at the focussed spot of $1.3 \times 1 \mathrm{~mm}^{2}$. Different wavelength ranges of the synchrotron radiation can be obtained using different windows, that is, $40-300 \mathrm{~nm}$ (without any window), 110-300 nm (with a LiF window) and 180-300 $\mathrm{nm}$ (with a quartz window). No difference in the processing was found in the different wavelength ranges.

The palladium deposit, after the substrate was washed in chloroform, was used for electroless plating of copper to check if enough Pd had been formed on the substrate surface.

\section{Spectrometric study of chemical changes}

At a low fluence level, namely $4 \mathrm{~mJ} \mathrm{~cm}^{-2}$, no palladium deposition could be induced from the palladium acetate spin-on film even after about

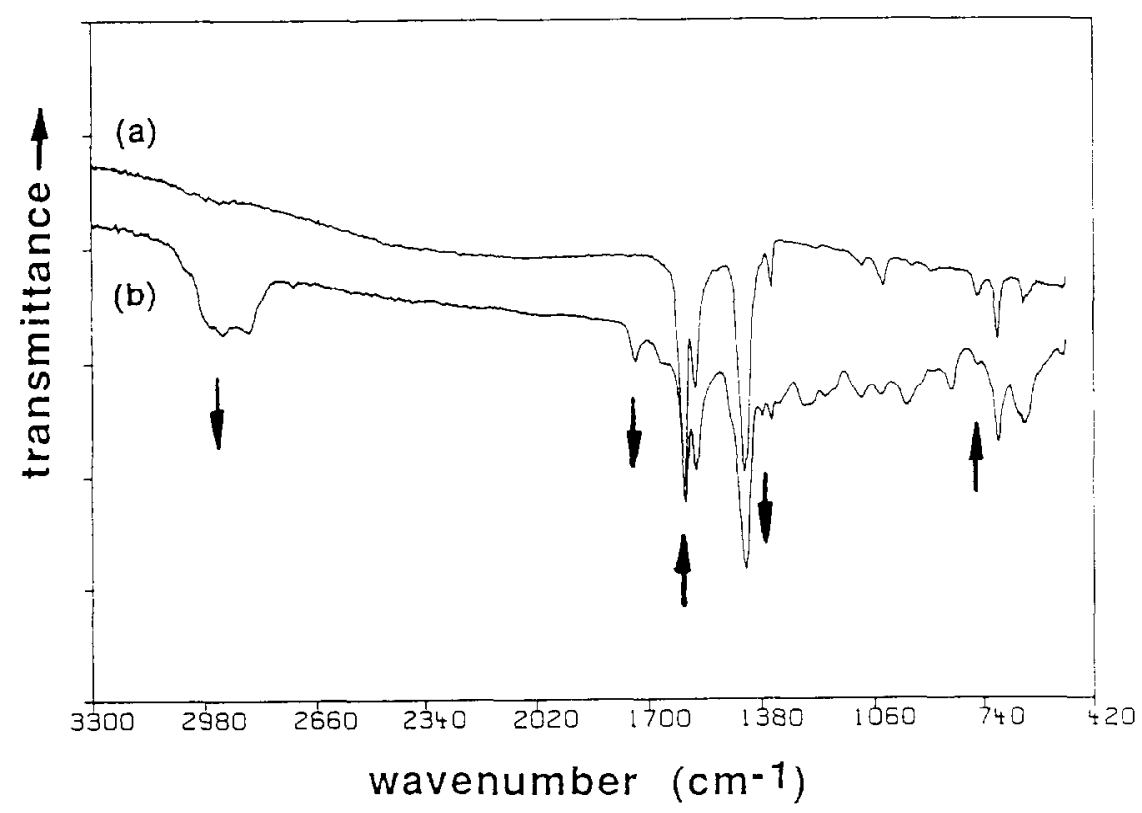

Fig. 1. FT-IR spectra $\left(420-3300 \mathrm{~cm}^{-1}\right)$ of the palladium acetate film before (a) and after (b) exposure to about thirty thousand shots of $248 \mathrm{~nm}$ laser pulses at a low fluence level $\left(4 \mathrm{~mJ} \mathrm{~cm}^{-2}\right)$. 
Table 2

Assignment of some changes of the IR spectral features in fig. 1

\begin{tabular}{llll}
\hline Wavenumber $\left(\mathrm{cm}^{-1}\right)$ & Vibration & Change & Meaning \\
\hline $2950-2800$ & $\mathrm{CH}$ stretching of $\mathrm{CH}_{3}$ & Increase & $\mathrm{C}-\mathrm{O}$ bonds break \\
1726 & $\mathrm{C}=\mathrm{O}$ stretching of ester & Appear & Pd-O bonds break \\
1596 & $\mathrm{C}=\mathrm{O}$ stretching of carboxylate & Decrease & Pd-O, $-\mathrm{O}$ bonds break \\
1370 & $\mathrm{CH}-\mathrm{O}$ deformation & Increase & $\mathrm{C}-\mathrm{O}$ bonds break \\
765 & $\mathrm{C}=\mathrm{O}$ scissoring of carboxylate & Decrease & Pd-O, C-O bonds break \\
\hline
\end{tabular}

thirty thousand shots of UV excimer laser pulses at $248 \mathrm{~nm}$ or $193 \mathrm{~nm}$. However, the chemical change in the film precursor after the irradiation can clearly be seen in the IR spectra shown in fig. 1 , where some changes of the spectral features (see arrows) are assigned [4] and summarized in table

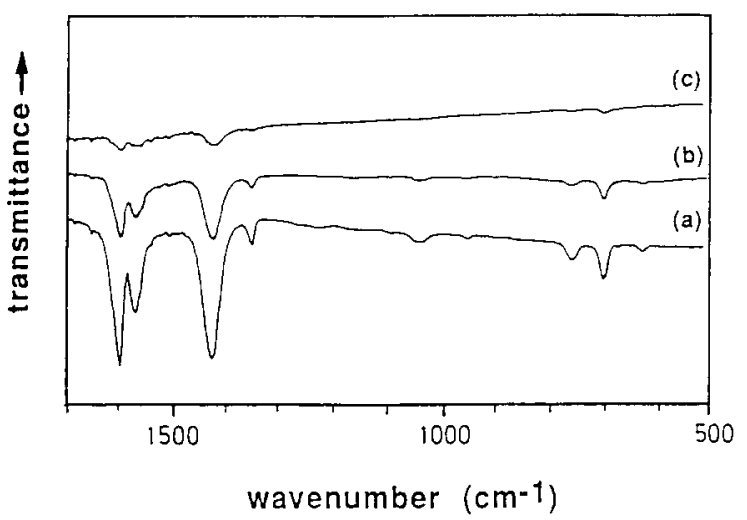

Fig. 2. FT-IR spectra $\left(500-1700 \mathrm{~cm}^{-1}\right)$ of the palladium acetate film before (a) and after exposure to four shots (b) and to thirty shots (c) of $248 \mathrm{~nm}$ laser pulses with $12 \mathrm{~mJ} \mathrm{~cm}^{-2}$.

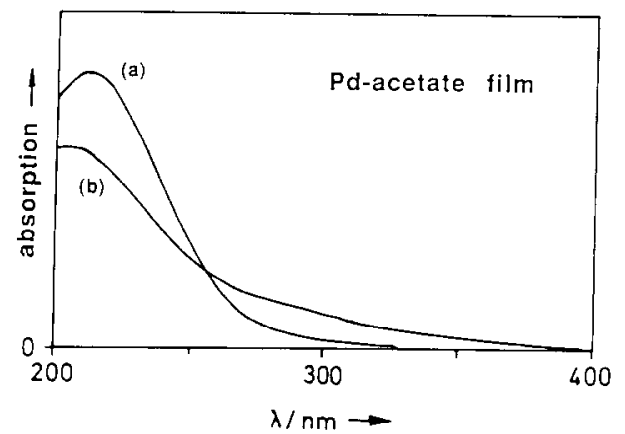

Fig. 3. UV spectra of the palladium acetate film before (a) and after (b) exposure to about thirty thousand shots of $248 \mathrm{~nm}$ laser pulses at a low fluence level $\left(4 \mathrm{~mJ} \mathrm{~cm}^{-2}\right)$.
2, which indicates that the palladium acetate film precursors have been dissociated by the UV laser light at 248 or $193 \mathrm{~nm}$ without sccing any film damage. The chemical changes under UV light are rather different from those under ion beam irradiation [5].

If the UV laser fluence was increased to above the prenucleation fluence threshold, the film was damaged and the palladium deposit appeared, which could be used for the copper plating. In this case, the spectral change in the IR spectra in fig. 2 hehaves completely differently from that in fig. 1. Here, the monotonous decrease in all the spectral features indicates that all the molecular photofrag-

Table 3

Ion signals detected due to desorbed photoproducts ${ }^{\text {a) }}$

\begin{tabular}{llllll}
\hline & \multicolumn{3}{c}{$m / e$} & & \\
\cline { 2 - 5 } & 59 & 44 & 43 & 27 & 15 \\
\hline Ions & $\mathrm{O}_{2} \mathrm{CCH}_{3}^{+}$ & $\mathrm{CO}_{2}^{+}$ & $\mathrm{OCCH}_{3}^{+}$ & $\mathrm{CCH}_{3}^{+}$ & $\mathrm{CH}_{3}^{+}$ \\
\hline a) The base pressure is $10^{-8}$ & mbar. & &
\end{tabular}

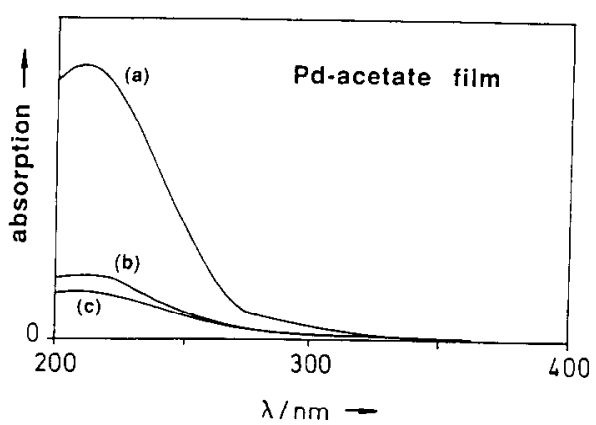

Fig. 4. UV spectra of the palladium acetate film before (a) and after exposure to four shots (b) and to thirty shots (c) of 248

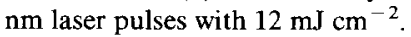


ments have been desorbed at a high laser fluence level, leading to the deposition of pure palladium. Actually, we measured the gas background by mass spectroscopy during the Pd-acetate film being exposed to $\mathrm{KrF}$ laser radiation above the fluence threshold for prenucleation and detected the desorbed photoproducts, as given in table 3. Our results in table 3 are somewhat different from those under ion-beam irradiation [6] but looks more reasonable. $\mathrm{CO}_{2}$ seems to be a main desorbed species and no Pd-containing ions at $\mathrm{m} / \mathrm{e}$ $>100$ were detected. Although the smaller mass ions like $\mathrm{CCH}_{3}^{+}$and $\mathrm{CH}_{3}^{+}$may also be due to further fragmentation of the larger mass ones, we can conclude from table 3 that the Pd-acetate film precursor has been decomposed by the $248 \mathrm{~nm}$ laser pulse, with the ligand part and its photofragments desorbed and the palladium deposited, which is consistent with the results concluded from the IR spectra (fig. 2).

For a cross-check, the changes in the UV absorption spectra were also measured below and above the fluence threshold, as shown in figs. 3 and 4. Here again, the spectral change is shown in fig. 3 (below the fluence threshold) while the monotonous decrease is shown in fig. 4 (above the threshold). These results agree with those from the IR spectra.

\section{Discussion}

From the above observations it is now clear that below the fluence threshold, UV laser-induced dissociation of the palladium acetate film occurs but no deposition of paliadium. This is similar to what has been found in the UV laser photoablation of organic polymers, namely the photolysis of the PMMA film before its photoablation, referred to as "incubation" [7]. Above the threshold, UV-laser-induced desorption of the ligand part photoproduct and its photofragments formed in the UV laser photolysis of the film precursors during a UV laser pulse results in $d e$ position of palladium as seen. Since this fluence threshold for the photodesorption process may exist [8], therefore, it is not surprising to find a fluence threshold for the UV excimer laser deposi- tion of palladium from Pd-acetate films. The prenucleaton threshold depends on the absorption coefficient of the UV radiation and the thickness of the film. When the laser fluence is increased much higher than the prenucleation threshold. photoablation rather than the deposition occurs. where not only the ligand part and its photofragments but also the palladium deposit, maybe even the film precursor molecules before their decomposition, will be desorbed. This photoablation has been known as "negative process" and used for application as well [9].

As studied above, the fluence threshold for the photodeposition is due to minimum amount of energy needed for the photodesorption. The broad-band UV or VUV light may reduce the threshold, due to efficient coupling of the photon energy into the film through its broad-band absorption. Therefore, the VUV synchrotron radiation $(40-300 \mathrm{~nm})$ with an average flux as low as $25 \mathrm{~mW} \mathrm{~cm}^{-2}$ was used for the deposition of palladium from Pd-acetate spin-on films. Since the $180-300 \mathrm{~nm}$ synchrotron radiation $\left(5 \times 10^{14}\right.$ photons $\mathrm{s}^{-1} \mathrm{~cm}^{-2}$ ) could be used to carry out the same processing on a transparent quartz substrate. no pyrolytic process should be involved. As shown in table 1 , the fluence threshold is considerably reduced by using the synchrotron radiation. More recently, it was found that the broad-band UV $\mathrm{Xe}_{2}^{*}$ excimer lamp $(166-178 \mathrm{~nm})$ with a low flux of $125 \mathrm{~mW} \mathrm{~cm}^{-2}$ can also be used for the same processing [10]. All these results confirm our above study.

\section{Conclusion}

A processing of thin palladium acetate spin-on films for electroless copper plating can be carried out using UV and VUV synchrotron radiation of an average flux of $5 \times 10^{15}$ photons $\mathrm{s}^{-1} \mathrm{~cm}^{-2}$. This confirms our mechanistic study, which shows that the UV photolysis of the palladium acetate film has occurred below the fluence threshold of the UV photodeposition and that this threshold is due to the desorption of the ligand and its photofragments leading to the deposition of palladium. 


\section{Acknowledgments}

The experiments were performed at BESSY, Berlin, Germany.

We would like to thank the staff of BESSY in Berlin for their unflagging expert help, Dr. J. Demny of ABB Heidelberg for his accurate surface diagnostic measurements and K. Müller for his technical assistance. Financial support by BMFT Nr. 05490 LA B9 (4.4-D21) is gratefully acknowledged.

\section{References}

[1] M.E. Gross, A. Appelbaum and P.K. Gallagher, J. Appl. Phys. 61 (1987) 1628.
[2] H.S. Cole, V.S. Liu, J.W. Rose, R. Guida, L.M. Levinson and H.R. Philipp. Proc. Electrochem. Soc. 88-10 (1988) 187.

[3] H. Esrom and G. Wahl, Chemtronics 4 (1989) 216.

[4] K. Nakanishi and P.H. Solomon, Infrared Absorption Spectroscopy (Holden-Day, San Francisco, CA, 1977).

[5] M.E. Gross, W.L. Brown, J. Linnros, L.R. Harriott, K.D. Cummings and H.O. Funsten, Mater. Res. Soc. Symp. Proc. 75 (1987) 91.

[6] M.E. Gross, W.L. Brown, L.R. I Iarriott, K.D. Cummings, J. Linnros and H. Funsten, J. Appl. Phys. 66 (1989) 1403.

[7] S. Küper and M. Stuke. Appl. Phys. A 49 (1989) 211.

[8] K. Domen and T.J. Chuang, Phys. Rev. Lett. 59 (1987) 1484.

[9] V.S. Liu and H.S. Cole, Chemtronics 4 (1989) 209.

[10] H. Esrom, J. Demny and U. Kogelschatz, Chemtronics 4 (1989) 202. 